\title{
A REVIEW REGARDING HAND EXOSKELETON TECHNOLOGIES FOR REHABILITATION
}

\author{
FL. BIROUAS \\ Department of Mechatronics, Faculty of Managerial and \\ Technological Engineering, University of Oradea \\ fbirouas@uoradea.ro \\ AR. NILGESZ \\ Department of Mechatronics, Faculty of Managerial and \\ Technological Engineering, University of Oradea \\ arnoldnilgesz@gmail.com
}

\begin{abstract}
This paper will be presenting a short review regarding robotic rehabilitation devices. The focus of rehabilitation are aimed for the human hand, mainly for regaining motor functions by the aid of robotics. A comprehensive statistical study will be presented regarding tendencies in the field of rehabilitation, medical robotics and technologies used for robotic exoskeletons based on existing published papers. A short review on existing practical examples is also presented. In the final part of the papers a short comparison is debated between soft robotic devices and rigid robotic devices used in hand rehabilitation. After presenting the review of the current state of the art a conclusion regarding the future direction of rehabilitation devices is proposed.
\end{abstract}

Keywords: Robotic Rehabilitation, Robotic Orthoses, Robotic Therapy, Hand Exoskeleton, Soft Robotics

\section{INTRODUCTION}

In this paper, a brief review will be presented of the current state of the art technology regarding hand motor function assistance and rehabilitation using robotic devices. Tendencies in this field of medical robotics will be observed and documented in a comprehensive statistical study. The purpose of this paper is to establish a good understanding of previous work in this field and determine the direction of future rehabilitation using robotic devices for hand motor function therapy and assistance.

Starting with the most rudimentary creations of man, tools, fire or agriculture, we have reached the present level, where we can benefit from technologies that were considered in the field of fiction a century ago. Although significant progress has been made in the industrial field of robotics [1], improving productivity and making hard labor obsolete, areas such as medical robotics, still need development and progress. [2]

It is well known that a significant part of the world's population suffers from at least one form of disability, either cognitive, emotional, sensory or motor impairment, and due to the a lack of access to advanced medical technology, the result is a reduced quality of life. Looking at the pyramid of needs described for the first time by Abraham Maslow in 1943, the physiological needs are the most important at the basis of our society. In an ideal world, the fundamental levels of physiological function should be part of our human rights where each person should have the fundamental right to life without disabilities.

The average life expectancy is steadily increasing due to the progress of science in medicine, chemistry and engineering. All these advances will bring a better living standard and also increase the average life of humans. This will in itself cause an unprecedented demographic change where a large proportion of the population will be composed of elderly people.

\author{
FL. AVRAM \\ Department of Mechatronics, Faculty of Managerial and \\ Technological Engineering, University of Oradea \\ favram@uoradea.ro \\ VL. MIHALCA \\ Department of Mechatronics, Faculty of Managerial and \\ Technological Engineering, University of Oradea \\ ovidiu@vmihalca.ro
}

It is an alarming sign for mankind that we must prepare ourselves scientifically to face the problems and diseases associated with the advanced age. Stroke is the leading cause of motor impairment in the world, and although paralysis after stroke is treatable via therapy, access to medical personnel to perform the therapy needed is often limited. If in the last century the first flight of a man to the moon has been reached, this century can be expected to bring achievements at the medical level, nanorobots, assistant robots, artificial organs, robotic exoskeletons, neural implants, etc.

\section{TENDENCIES IN HAND MOTOR FUNCTION REHABILITATION}

In the field of medical robotics, the robot design has to adapt to the challenges of the living world, in this sense certain scientific branches such as bionics and biomechanics have become a subject of interest, exploding in the last 20 years with significant innovations.

Applications where the human body is augmented with biomechanical or bionic devices are numerous, they can be delimited in two major branches: internal and external, the study in this paper will focus on the external ones. The external augmentation of the human body can be delimited in two large branches, namely: prostheses and orthoses.

This paper main focusing is on robotic orthoses and the technologies that are used in robotic rehabilitation devices for hand motor function rehabilitation therapy.

In order to be able to represent the progress in this area of research, a statistical study was elaborated based on a series of technical terms encountered in the scientific literature of this field. The statistics include works from 1994 to 2017. The study data was collected from the online Science Direct database. ScienceDirect contains a vast database of scientific, medical, economic research and beyond. This database hosts over 12 million articles from over 3,500 academic journals.

The following terms have been chosen in this study: Rehabilitation, Robotic Rehabilitation, Stroke Rehabilitation, Orthoses, Biomechanics [3], Bionics [4], Robotic Orthoses, Exoskeleton, Robotic Exoskeleton, Hand Exoskeleton, Soft Robotics and Soft Actuator.

The collected data generated is shown in table 1, which represents the number of published scientific papers referring to the terms analyzed.

It can be seen in the graph of figure 1 that biomechanics, soft robotics, and soft actuators are among the most used keywords in the scientific works of the last 20 years. Taking into account the large number of works that refer to 
biomechanics, soft robotics, and soft actuators, the following statistics that refer to key keywords with a narrower area will not be directly compared to these terms.

Table 1. RELATIONSHIP BETWEEN THE KEYWORDS SEARCHED AND THE NUMBER OF PUBLISHED PAPERS CONTAINING THOSE TERMS

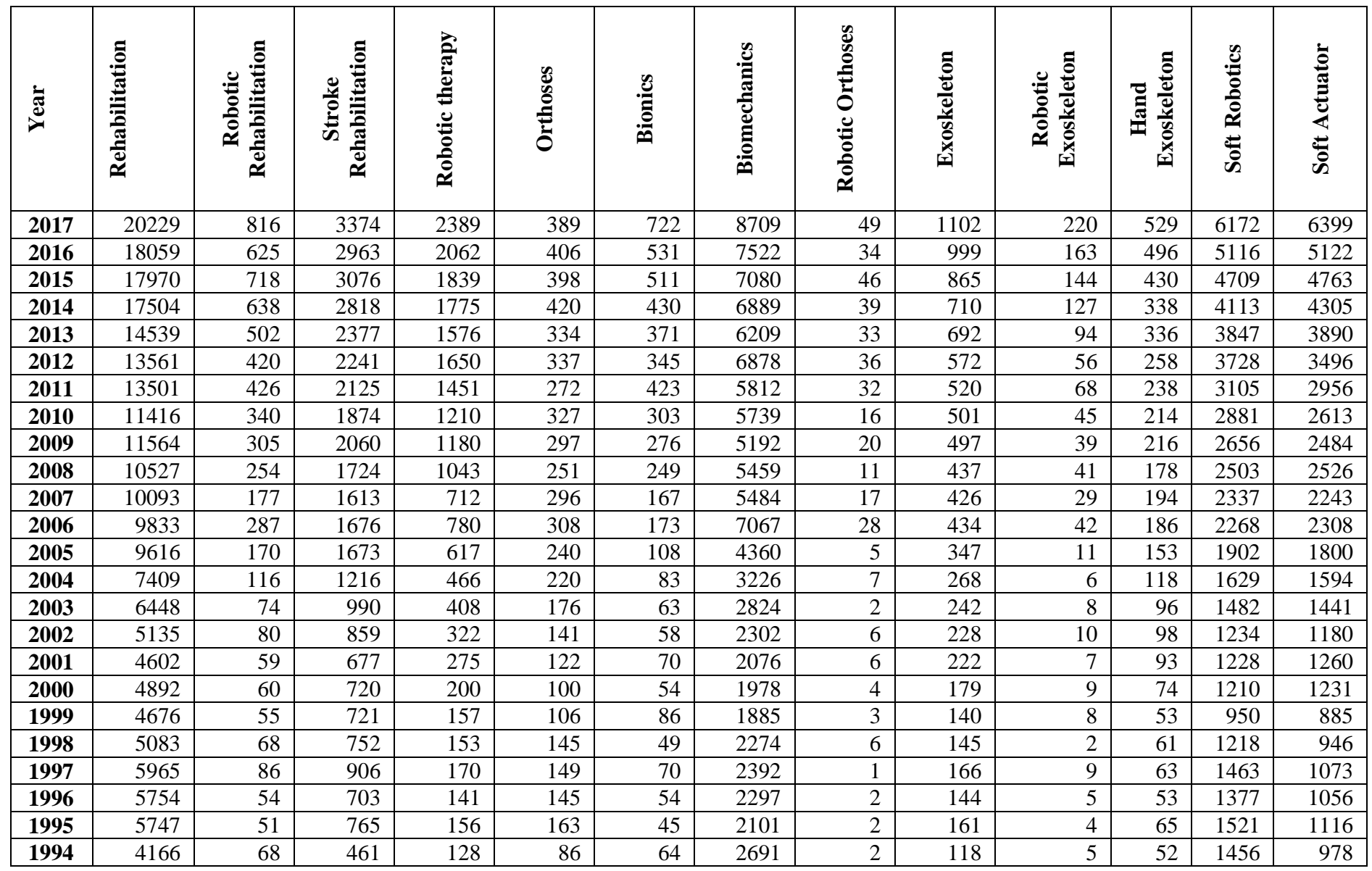

Observing the graph in figure 2, we see that the term hand exoskeleton has been more and more common in scientific works, and more so has become a term used more often than the orthoses term from 2015 to the present.

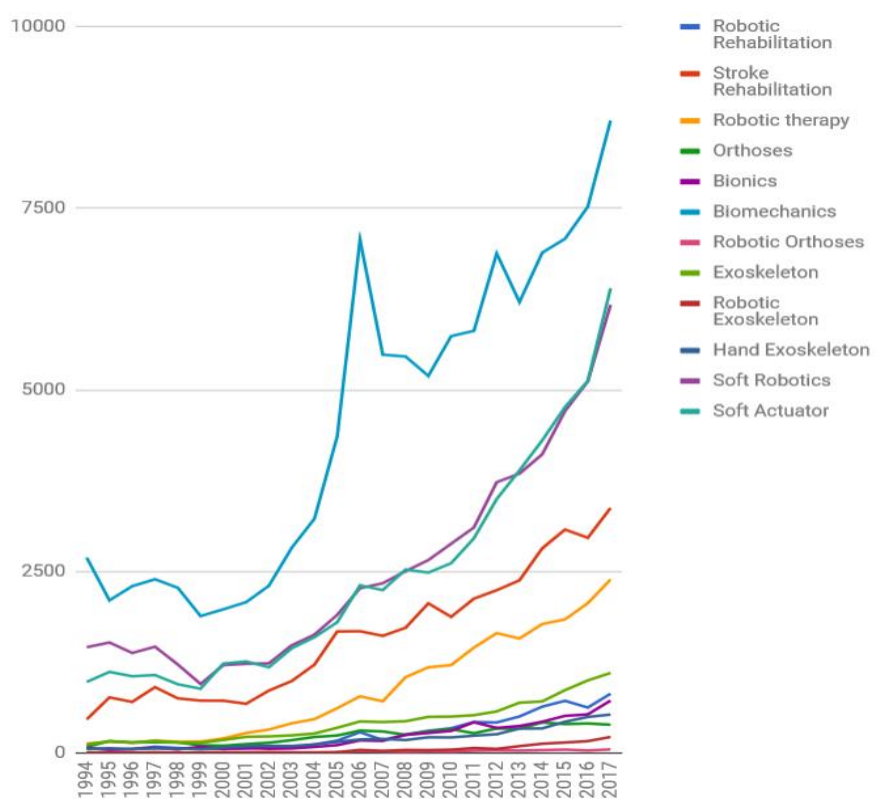

Fig. 1 Analysis between the searched keywords and the number of published papers containing those terms
Also in this graph we can see that in 2005 there was significant growth in the use of terms such as Robotic Orthoses and Robotic Exoskeleton. We can say that during this period a new trend in medical robotics has begun if we take into account the small number of works that refer to these terms before 2005 .

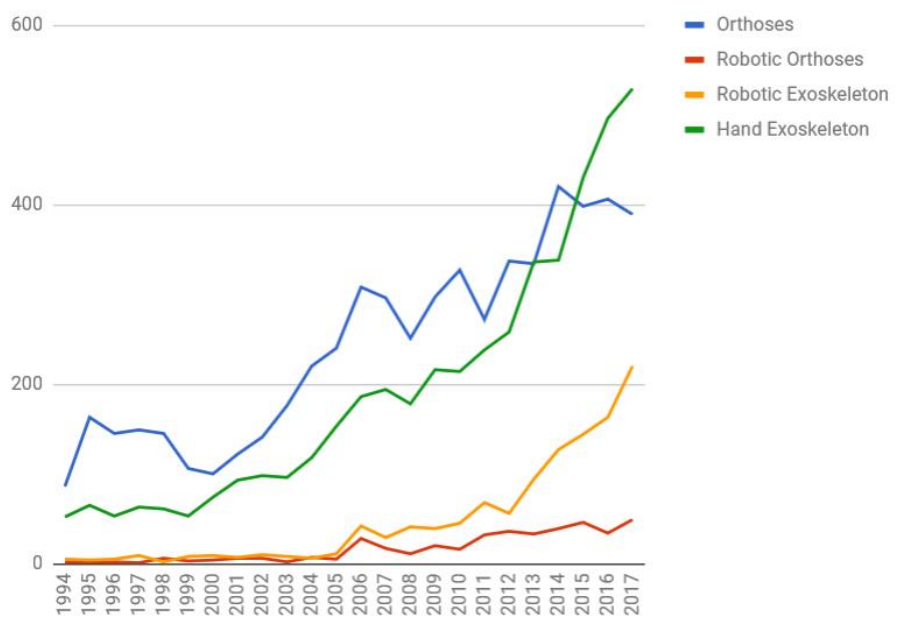

Fig. 2 Keyword report: orthosis, robotic orthosis, robotic exoskeleton and hand exoskeleton 
This study also took into account the progress in the use of key words such as robotic rehabilitation, stroke rehabilitation and robotic therapy. The graphical representation can be seen in figure 3. As in the previous graph, we can see an exponential growth, in this case beginning approximately in 2001. This indicates an increased interest in solving problems associated with medical rehabilitation using robotic devices.

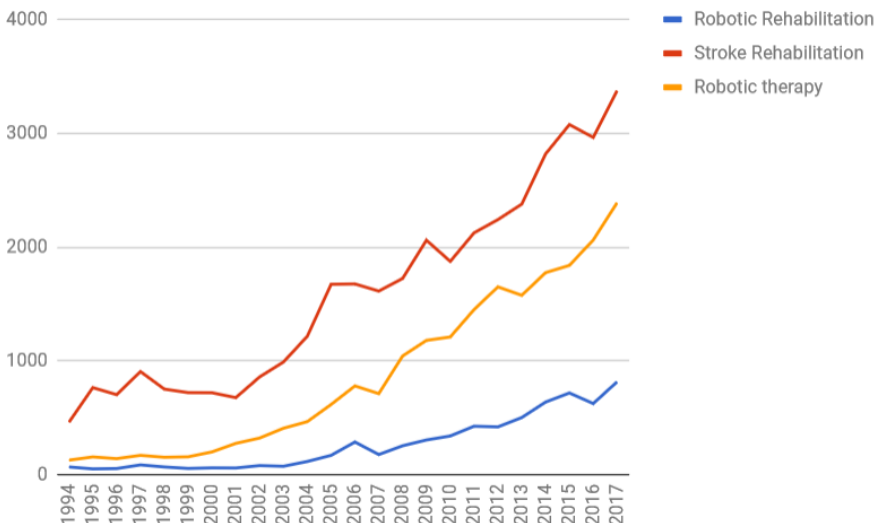

Fig. 4 Keyword report: robotic rehabilitation, stroke rehabilitation accident and robotic therapy

The graph depicted in figure 5 illustrates the progress of the use of Soft Robotics and Soft Actuators relative to the terms Stroke Rehabilitation. It can be seen that there is significant progress in both the branches of Soft robotics, and rehabilitation after a cardiovascular accident. We can say that there is a close link between the advances in the rehabilitation technique between the terms mentioned. Many of the medical rehabilitation applications using robotic exoskeletons are based on unconventional actuation modes such as actuators that mimic artificial muscles. Actuators that are part of the Soft robotics and Soft actuators.

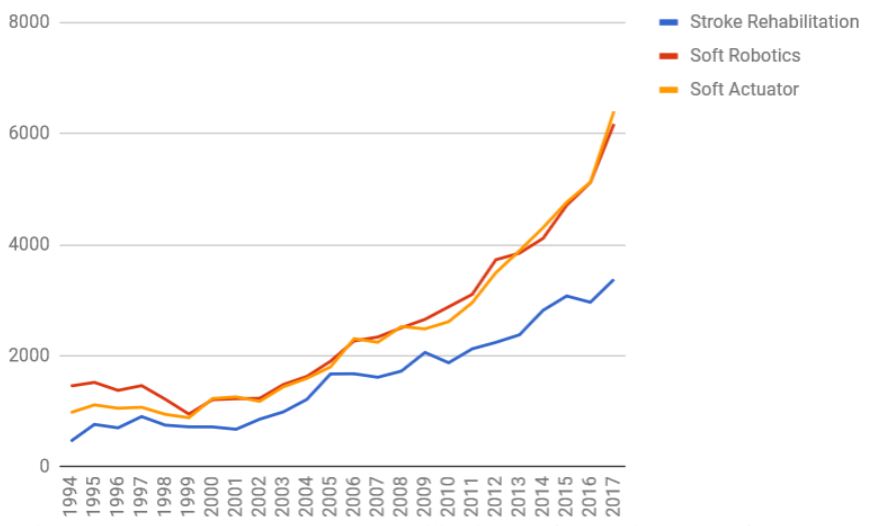

Fig. 5 Keyword report: Stroke rehabilitation, soft robotics and soft actuators

\section{EXISTING HAND EXOSKELETON}

In the literature there is a multitude of approaches to solving the problems of transmitting motion from the actuator to the fingers of the patient, as seen in figure 6 . These approaches are reviewed and presented in this chapter in order to have a broader understanding of the limitations achieved by the present state.

In elaborating this comparison, we will consider the most significant works on this branch of medical robotics, with particular emphasis on works with practical achievements of the authors. The benefits and, implicitly, the disadvantages of each approach will be emphasized.

First of all, robotic hand exoskeleton can be grouped into two distinct groups, those with a rigid construction, that have a structure of the exoskeleton made of rigid solids but articulated by the nature of the assembly, and those having a flexible construction made of deformable and elastic materials, found in the literature in the field of Soft Robotics and Soft Actuators.

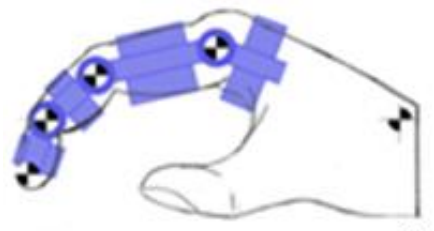

a)
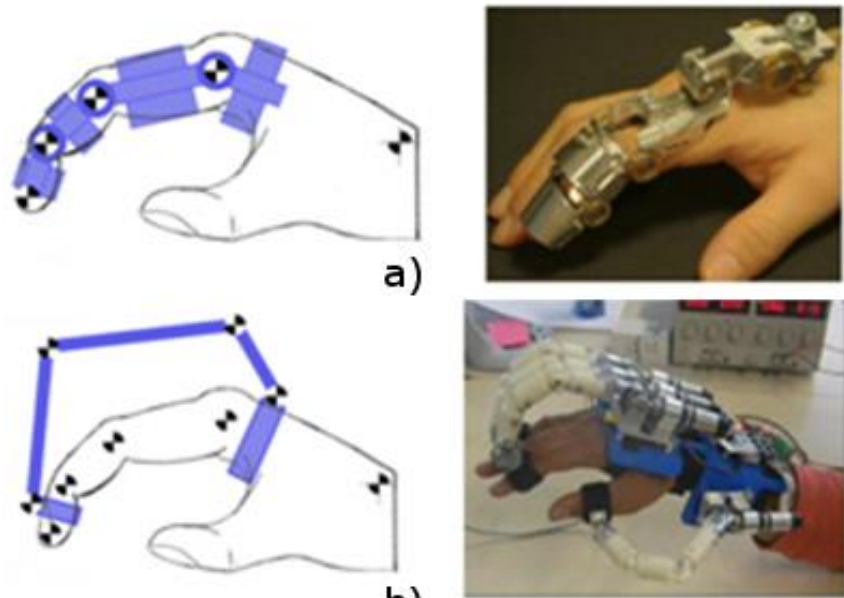

b)
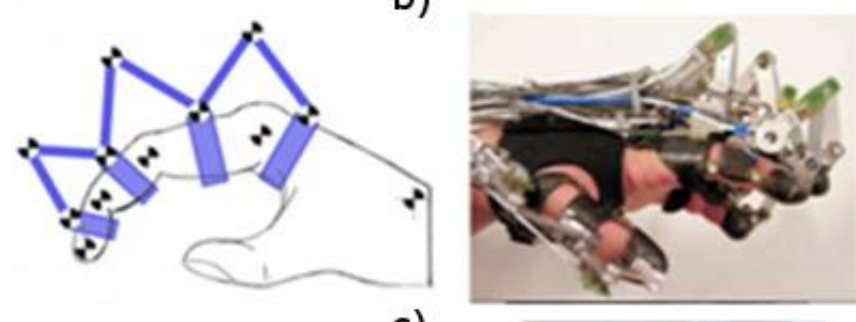

c)
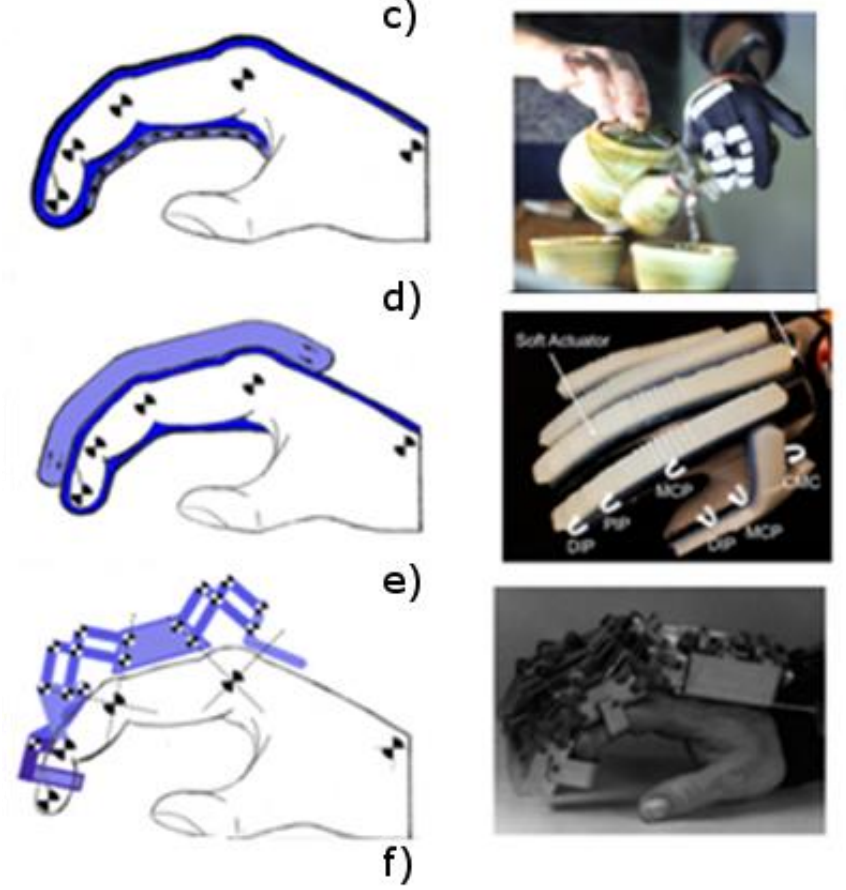

Fig. 6 Constructive types of existing robotic exoskeletons [5] [6] [7] [8] [9] [10]

a) Handexos

Described as an robotic exoskeleton-type application for aerospace use was developed by A. Chiri, F. Giovacchini, S. Et al in the ARTS Laboratory, Scuola Superiore Sant 'Anna. The assembly was designed for all five fingers, each finger being an independently controlled module. HANDEXOS is composed of a rigid structure that attaches to the human arm. The movement of the fingers is made of a series of rotation and translation rods. Unlike some other examples, this application uses a passive translation that is designed to follow the contour 
of the finger to flexion and extension. At the same time, the inside of the exoskeleton is designed to include a conformable material that adapts to the anatomical forms of the finger of the wearer.

The movement of each finger consists of three rotation spindles, six pulleys, each couple having two pulleys. These rotation spindles help to flex and extend the fingers, namely DIP, PIP and MCP joints. [5]

Each finger module is composed of 3 links for the phalanges, where the center of rotation of each connection is matched with the corresponding joint of the human finger. The flexion and extension of the MCP joint is driven by a slidercrank-like mechanism, while the PIP and DIP joints are driven by Bowden cable transmissions. The 3 joints of each finger are underactuated because they are driven using a single actuator unit. For the finger module, 3 force sensors are mounted on the surface of the inner side of each of the three palmar shells to sense the interaction force. The linear slider for MCP rotation is equipped with strain gauges to measure the force transmitted by the driving cable. [7]

\section{b) Hexosys}

Iqbal et al. proposed the system EXOskeleton SYStem (HEXOSYS), which at the first prototype had two finger for rehabilitation, and then at the second iteration all 5 fingers (which can be seen in figure 6. b). [6] Each finger is driven by using an underactuated linkage driven by an electric motor. The linkage structure adopted in this device is a three-link planar underactuated mechanism having a single attachment point. A custom-made force sensor is integrated into the connecting link. [11]

\section{c) Wege A And Hommel G}

A practical example was developed by Wege $\mathrm{A}$ and Hommel G. This can be seen in Figure 6. C. In this application, each finger has four degrees of freedom, namely flexion and extension for the MCP, PIP and DIP plus abduction / adduction of the MCP joint. Fingers by construction can be bidirectionally operated on all joints. [8]

The hand exoskeleton actuates each joint via a Bowden cable driven by an electric motor. Bidirectional movement is supported by the use of two pull cables for each joint, diverted by a pulley on both ends. Only one motor is used for each joint, which introduces some slackness when compared to a solution using one motor for each direction. The motion is applied through a leverage construction on each finger attachment.

This device is controlled by EMG signals. Each finger rests in its relaxed position when no muscle activation is measured. Depending on the muscle activation, a linear force is calculated and the fingers are moved as if acting against a constant friction. The movements of the MCP, PIP, and DIP joints are performed in a coupled motion. [7]

\section{d) Exo-Glove - Hyunki In Et Al.}

The glove-type hand exoskeleton developed by Hyunki In, Brian Byunghyun Kang, Minki Sin, and Kyu-Jin Cho. In seen in figure 6. d) was designed to assist disabled people. This device adopts an underactuated cable-driven mechanism attached to a glove. Because there is no rigid linkage, the wearer's hand becomes the linkage structure for operation of the exoskeleton. A cable exerts a flexion force on each finger, while the extension force is provided passively by a spring. All of the actuated fingers are driven by a single motor. However, the tendon excursions which occur during the finger movements are different for each finger because of the differences in the moment arms. There are therefore stacked pulleys with different diameters at the output shaft of the motor, providing suitable amounts of tendon excursion for each finger. An electromyography (EMG) signal is used to control the device in a simple on-off manner. The device exerts a flexion force when the EMG signal exceeds a predefined threshold. [9]

The concept of force transmission on the finger is very similar to the biological tendons in the human hand, but instead of the biological tendons the wires are driven and guided into strategically placed channels. Applications are mainly limited to rehabilitation, easy handling and recovery.

\section{e) Exoglove - Hong Kai. Yap}

Given the limitations of rigid constructional devices, various soft devices have been proposed, such as pneumatic elastomeric actuators that offer very good compliance with the patient's hand. A practical example was developed by Hong Kai. Yap, Jeong Hoon. Lim, Fatima. Nasrallah, James C. H. Goh, and Raye C. H. Yeow. [10] seen in figure 6. e).

From a technical point of view, more elaborate development work is needed than the tendon drive mechanism and also more expensive to manufacture. For the same reason as the previous type of construction, this type is also used mainly in rehabilitation applications.

This constructive solution has a multitude of advantages, one of which is that, by the soft nature of the exoskeleton, the chances of accidents or damage caused by the exoskeleton are minimal compared to a rigid constructive solution. The cost is also considerably lower due to the fact that it does not use expensive components and is relatively easy to manufacture.

The exoskeleton consists of a textile glove on which there is a velcro in the attachment areas of the pneumatic elastomeric actuator. The actuator can be manufactured to produce deformations in some queens, with areas where bending and other areas remain straight to the flexing movement.

\section{f) Space Suit Glove - Shields}

One of the first examples of hand robotic exoskeleton in literature and published in 1997, Shields, B. L., et al., developed a robotic hand exoskeleton for aerospace applications.

Space suits and gloves are stiffened by the pressure difference when they are exposed to the vacuum of space during extravehicular activities (EVA). Because it is difficult for astronauts to move against this stiffness, space suits have caused reduced dexterity and increased fatigue. To overcome this problem, some devices have been developed. Shields et al. proposed a hand exoskeleton for an EVA glove. It has three actuated fingers (index, middle, ring-small), with one DOF for each finger. The links for each finger form four-bar mechanisms to allow the joints to rotate about remote centers that are coincident with the joints of the wearer's fingers. The motions of the two joints for each finger are coupled together.

This device exerts a flexion force generated by motors via a cable-driven cam mechanism, while the extension is performed using a passive force provided by the stiffness of the space suit glove. The user's intention to flex the glove is sensed by force sensors mounted inside each fingertip. The control of the device is performed in a simple on/off manner with two threshold levels that classify the operation modes into flexion, stop, and extension modes. [11] 


\section{A. Exploration of developments on the rigid robotic exoskeleton branch}

There are a considerable number of papers dealing with rigid mechanical solutions. This is among the first examples of literature applications in this field. Device design is based on non-deformable solid components assembled either with joints that are concentric with the rotation of the finger of the finger or placed at points different from the axis of rotation of the rotation of the finger of the finger. Bearings and sliding bearings are used to articulate the assemblies. The attachment of the hand exoskeleton and the movement of the actuator to the finger is done mechanically using rigid parts.

\section{B. Exploring developments on the flexible robotic exoskeleton branch}

A trend can be observed that flexible exoskeletons apply usually unconventional solution; the technology developed or used in the realization of these exoskeletons often mimics biological structures such as muscles and tendons. Also, these applications often find their place in the field of medical robotics, because their flexible nature offers a high degree of compliance with the human body interface.

The materials used to make these robotic devices are often silicone or textile materials and, in more recent cases, intelligent flexible materials. These devices are of great interest in medical robotics due to the huge potential of applications and utilities. [12] [13]

In the realization of these devices, manufacturing techniques found in the field of unconventional technologies are used, classical actuators such as electric, pneumatic or hydraulic motors do not find their place in this field, instead a multitude of unconventional actuators such as artificial muscles constructed from flexible materials such as silicone and / or in combinations with textile materials are used. The control of these actuators is based on compressed air, fluid, thermal expansion or other phenomena that cause movement.

Although progress has been made on several fronts by independent authors, the trend seems to be that medical robotics will increasingly be based on innovations in the field of Soft Robotics and Soft Actuator. Man is a biological being and augmenting our body using robotic technology will also be with the aid of devices that mimic biological properties. [14]

Ergonomically, it is more beneficial for an element attached to a patient to be rehabilitated or assisted to be soft and not rigid. Real example by analogy we can take modern shoes, even after centuries of technological improvements in the field of materials, design and manufacture, we have not yet managed to completely eliminate their discomfort. If even shoes that have improved flexibility and rigidity for the human foot cause discomfort, an exoskeleton ridge made of metallic and rigid polymers will implicitly cause discomfort after a certain period of use.

\section{CONCLUSIONS}

Following the study of the current state of robotic hand rehabilitation devices, it can be said that there is significant progress, and that applications have improved over the years, each author coming up with contributions and innovations, resolving existing problems and coming up with new solutions.

In both the medical, industrial, aerospace and military fields, robotic mechanisms are developed to directly improve human life, and in the years to come we will see increasing growth in the complexity and efficiency of these applications. From what we have seen, it can be said that the robotic exoskeletons of the future will be hybrids, composed of both rigid and flexible materials, intelligent materials with properties closer to living organisms.

\section{Acknowledgement}

$\mathrm{PhD}$ School of Engineering Sciences - University of Oradea

\section{References}

1. Tarcă, Cătălin Radu. ADVANCED MECHATRONICS. Debrecen : University of Debrecen, 2012. ISBN 978-963-473-508-3.

2. Cătălin, Țarcă Radu. Introducere in Robotica. s.l. : Editura Universitătii din Oradea. ISBN 973-613-456-3.

3. Biomechanicswikipediahttps://en.wikipedia.org/wiki/Biomechanics

4. Bionics. wikipedia. [Online] [Cited: 19 Februarie 2018.$]$ https://en.wikipedia.org/wiki/Bionics.

5. Handexos towards a support device for hand activities and telepresence. A. Chiri, F. Giovacchini, S. Roccella, N. Vitiello, E. Cattin, F. Vecchi. s.1. : ESTEC, European Space Agency, 2008, Proceedings of the 10th Workshop on Advanced Space Technologies for Robotics and Automation.

6. Stroke rehabilitation using exoskeleton-based robotic exercisers Mini Review. Jamshed Iqbal*, Khelifa Baizid. 2015, Biomedical Research, Vol. 26, pp. 197-201. ISSN 0970-938X.

7. Current Hand Exoskeleton Technologies for Rehabilitation and Assistive Engineering. Pilwon Heo1, Gwang Min Gu1, Soo-jin Lee2, Kyehan Rhee2 and Jung Kim1. 5, Mai 2012, INTERNATIONAL JOURNAL OF PRECISION ENGINEERING AND MANUFACTURING, Vol. 13, pp. 807-824. DOI: 10.1007/s12541-012-0107-2.

8. Mechanical Design and Motion Control of a Hand Exoskeleton for Rehabilitation. Andreas Wege, Konstantin Kondak, Günter Hommel. s.l. : IEEE, 2005. Proceedings of the IEEE International Conference on Mechatronics and Automation. Vol. 1, pp. 155 - 159.

9. Exo-Glove: A Soft Wearable Robot for the Hand with a Soft Tendon Routing System. Hyunki In, Brian Byunghyun Kang, Minki Sin, and Kyu-Jin Cho. 1, Martie 2015, IEEE Robotics \& Automation Magazine, Vol. 22, pp. 97 - 105. 10.1109/MRA.2014.2362863.

10. A Soft Exoskeleton for Hand Assistive and Rehabilitation Application using Pneumatic Actuators with Variable Stiffness. Hong Kai. Yap, Jeong Hoon. Lim, Fatima. Nasrallah, James C. H. Goh, and Raye C. H. Yeow. Washington : s.n., 26-30 Mai 2015, International Conference on Robotics and Automation (ICRA), pp. 4967-4972.

11. Current Hand Exoskeleton Technologies for Rehabilitation and Assistive Engineering. P. Heo, G. Min Gu, S. Lee, K. Rhee and J. Kim,. 5, Mai 2012, INTERNATIONAL JOURNAL OF PRECISION ENGINEERING AND MANUFACTURING, Vol. 13, pp. 807-824.

12. Review of manufacturing processes for soft biomimetic robots. Cho, Kyu-Jin, et al. 3, 11 Octombrie 2009, International Journal of Precision Engineering and Manufacturing, Vol. 10, pp. 171-181.

13. Soft Robotics: Biological Inspiration, State of the Art, and Future Research. Trivedi, D., Rahn, C. D., Kier, W. M., \& Walker, I. D. 3, 2008, Applied Bionics and Biomechanics, Vol. 5, pp. 99-117.

14. Design, fabrication and control of soft robots. Rus, Daniela and Tolley, Michael T. s.l. : Nature Publishing Group, 27 Mai 2015, Nature 521, pp. 467-475. 